
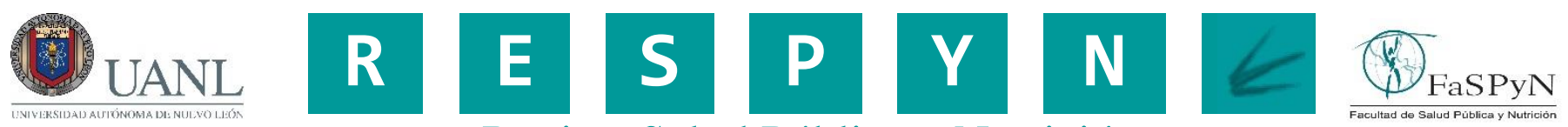

Revista Salud Pública y Nutrición

\title{
ÍNDICE DE MASA CORPORAL Y RENDIMIENTO ACADÉMICO EN ESTUDIANTES UNIVERSITARIOS.
}

\author{
BODY MASS INDEX AND ACADEMIC PERFORMANCE IN UNIVERSITY STUDENTS
}

Landeros Ramírez Patricia ${ }^{1}$, Gómez Cruz Zoila ${ }^{1}$, Rimoldi Rentería Ma. de Jesús², Parada Barrera Gloria², Núñez-Hernández Alfonsina ${ }^{1}$.

1 Universidad de Guadalajara, Departamento de Salud Pública, México. 2 Universidad de Guadalajara, Departamento de Ciencias Ambientales, México.

Citation: Landeros Ramírez P., Gómez Cruz Z., Rimoldi Rentería M.J., Parada Barrera G., Núñez-Hernández A. (2018) Índice de Masa Corporal y Rendimiento Académico en Estudiantes Universitarios. Revista de Salud Pública y Nutrición, 17(4), 34-40.

Editor: Esteban G. Ramos Peña, Dr. CS., Universidad Autónoma de Nuevo León, Facultad de Salud Pública y Nutrición, Monterrey Nuevo León, México. Copyright: (02018 Landeros Ramirez P. et al. This is an open-access article distributed under the terms of Creative Commons Attribution License [CC BY 4.0], which permits unrestricted use, distribution, and reproduction in any medium, provided the original author and source are credited.

Competing interests: The authors have declared that no competing interests exist.

DOI: https://doi.org/10.29105/respyn17.4-4

Recibido: 17 de agosto 2018; $\quad$ Aceptado: 06 de diciembre 2018

Email: patricia.landeros@academicos.udg.mx 


\title{
ÍNDICE DE MASA CORPORAL Y RENDIMIENTO ACADÉMICO EN ESTUDIANTES UNIVERSITARIOS
}

\author{
Landeros Ramírez Patricia ${ }^{1}$, Gómez Cruz Zoila ${ }^{1}$, Rimoldi Rentería Ma. de Jesús², Parada Barrera Gloria², Núñez- \\ Hernández Alfonsina ${ }^{1}$.
}

1 Universidad de Guadalajara, Departamento de Salud Pública. 2 Universidad de Guadalajara, Departamento de Ciencias Ambientales.

\section{RESUMEN}

Introducción: Los estudiantes universitarios son un grupo vulnerable que se encuentra en proceso de formación, con hábitos alimentarios en muchos casos no saludables que pueden afectar el rendimiento académico. Objetivo: Identificar la relación entre el Índice de Masa Corporal (IMC) y el rendimiento académico de los alumnos del Centro Universitario de Ciencias Biológicas y Agropecuarias de la Universidad de Guadalajara. Material y Métodos: Es un estudio de casos y controles, para lo cual se tomó peso y estatura de los estudiantes y se calculó el IMC, se formaron dos grupos: casos (estudiantes con bajo peso, sobrepeso y obesidad) y controles (normopeso). El rendimiento académico fue clasificado en tres categorías: Excelente/Muy Bueno (90-100), Bueno (80-89) y Regular/Malo (<80), de acuerdo a su situación académica se catalogaron como: titulados, egresados, activos y estudiantes irregulares. El análisis estadístico se realizó mediante el coeficiente de correlación de Pearson, pruebas de media y desviación estándar y prueba t de student. Resultados: Participaron 200 alumnos, con edad promedio de 21 años, 97 casos ( $13 \%$ tuvieron bajo peso, $26.5 \%$ sobrepeso y $9 \%$ obesidad) y 103 controles (51.5\%). El $40.5 \%$ de alumnos con sobrepeso presentaron rendimiento académico significativamente menor $(p<0.05)$ en relación al $13.5 \%$ con normopeso. Conclusiones: En esta muestra de estudio se encontró una correlación negativa y significativa entre el IMC y el promedio académico.

Palabras Clave: Índice de Masa Corporal (IMC), rendimiento académico, estudiantes universitarios.

\section{ABSTRACT}

Introduction: The university students are a vulnerable group in the sense of their eating habits that in many cases are unhealthy with impact on their academic performance. Objective: Identify the relationship between the Body Mass Index (BMI) and the academic performance of the students from of the University Center of Biological and Agricultural Sciences of the University of Guadalajara. Methods: A study of cases and controls, the student's weight and height were taken into consideration and their BMI was calculated. Two groups were considered: Group cases (underweight, overweight and obesity students) and Group controls (normal weight students). The academic performance was classified into three categories: Excellent/Very Good (90-100), Good (80-89) and Regular/Bad (<80), according to their Academic Situation the students were cataloged as: certified professionals, graduates, active and non-regular students. The results were statistically analyzed under the Pearson correlation coefficient, mean, standard deviation tests and student t test. Results: From 200 students analyzed, with an average age of 21 years old, 97 were classified into the Group Cases (13\% were underweight, 26.5\% overweight and $9 \%$ obese) and 103 into the Group Controls (51.5\%). Concerning the Academic Performance, 40.5\% of overweight students showed significantly lower academic performance $(p<0.05)$ in comparison to $13.5 \%$ with normal weight. Conclusions: The results of this study showed a negative and significant correlation between the BMI and the academic average.

Key words: Body Mass Index (BMI), academic performance, university students. 


\section{Introducción}

Entre los grupos etarios claves para establecer estilos de vida saludable se encuentran los jóvenes universitarios quienes adquieren hábitos que en la mayoría de los casos se mantienen en la edad adulta, entre los que destaca una inadecuada calidad de la alimentación, si éstos permanecen se convertirán en factores de riesgo para la salud (Sánchez-Ojeda y De Luna-Bertos, 2015). El ingreso de los jóvenes a la universidad es una etapa crítica, los cambios en los métodos de aprendizaje y el interés por obtener mejores resultados académicos les crean conflictos emocionales que pueden contribuir en la sensación de hambre, apetito y saciedad e influir en la selección adecuada de alimentos que les permita obtener más energía para cumplir con los deberes encomendados (Fleitas, García y Zambrano, 2015).

Respecto al rendimiento académico, este es considerado como la suma de diferentes factores que desarrolla y aplica la persona que aprende, atribuyéndole un valor al logro que el estudiante ha alcanzado en las tareas académicas, el cual se mide mediante las calificaciones obtenidas, ya sea con un valor cuantitativo o cualitativo, cuyos resultados integran las materias aprobadas o reprobadas, el grado de éxito académico, o la deserción (Jiménez y Madrigal, 2009).

El mecanismo por el cual la salud y la nutrición influyen en los logros educativos no está bien establecido, pero sí se conoce que la mala salud y la malnutrición en la primera infancia pueden afectar las capacidades cognitivas, alterando el proceso de aprendizaje y consecuentemente el logro educativo (Ghosh, Rakshit y Bhattacharya, 2013).

En México, el estudio realizado por García, Padrón, Ortiz-Hernández, Camacho y Vargas (2005), muestra la notoria influencia de una nutrición deficiente en escolares respecto a su rendimiento académico. Jiménez y Madrigal (2009), encontraron que el $38 \%$ de los jóvenes con sobrepeso y obesidad mostraban bajo rendimiento académico. Al respecto, Jukes, McGuire, Method y Sternberg (2002), señalan que existe evidencia sobre la importancia de la nutrición como elemento esencial en el aprendizaje y rendimiento académico, lo cual repercute a mediano y largo plazo en una mayor productividad y mejores salarios. A nivel nacional la prevalencia de sobrepeso y obesidad en adultos ha aumentado de manera importante en los últimos años, en la actualidad alcanza el $72.5 \%$ de la población según datos reportados por la Encuesta Nacional de Salud y Nutrición Medio Camino (ENSANUT MC, 2016).

Diversos autores mencionan que los estudiantes universitarios son un grupo vulnerable desde el punto de vista nutricional, ya que se caracteriza por omitir comidas con frecuencia y tener preferencia por comida rápida (Antonella, Vidal, Brassesco, Viola y Aballay, 2015; Arroyo et al., 2006). Con el fin de conocer la situación particular en nuestro medio educativo es pertinente abordar esta problemática que podría estar incidiendo en la formación académica y por ende en el aspecto laboral futuro, por lo que es importante determinar si existe relación entre el Índice de Masa Corporal (IMC) y el rendimiento académico de los alumnos del Centro Universitario de Ciencias Biológicas y Agropecuarias (CUCBA), lo que permitirá fundamentar propuestas tendientes a generar $\mathrm{y}$ fortalecer estilos de vida saludables en la comunidad universitaria y con ello contribuir a mejorar los indicadores educativos del Centro Universitario..

\section{Material y Métodos}

El presente es un estudio de casos y controles que se llevó a cabo en la población estudiantil de las licenciaturas de Agronomía, Biología, Ciencia de los Alimentos y Medicina Veterinaria y Zootecnia del Centro Universitario de Ciencias Biológicas y Agropecuarias (CUCBA) de la Universidad de Guadalajara, conformado por 200 alumnos de ambos sexos, con edades entre 18 y 27 años, a quienes se les explicó el propósito del estudio y se obtuvo su consentimiento informado para aplicar el instrumento de evaluación. Para determinar el tamaño de la muestra se consideró una investigación previa en este Centro, donde los resultados mostraron que de acuerdo al IMC el 39.9\% $(n=133)$ de los estudiantes presentaron un IMC alterado (bajo peso: $11.5 \%$, sobrepeso: $20.5 \%$ y obesidad: $8.3 \%$ ), considerándose éstos como el grupo de casos para el presente trabajo, de los cuales 97 estudiantes fueron incluidos y 36 excluidos por no presentar datos completos. El protocolo de estudio fue aprobado por el Colegio Departamental del Departamento de Salud Pública y la Coordinación de Investigación del Centro Universitario. 
A cada uno de los alumnos participantes en el estudio se les tomó peso y estatura, y con estos datos se determinó el Índice de Masa Corporal (IMC), el peso fue medido utilizando una báscula calibrada marca Seca Modelo 700 con una precisión de 100 g, el IMC se calculó utilizando la siguiente ecuación: peso en $\mathrm{kg} /$ talla2 y se clasificaron de acuerdo a los criterios de la Organización Mundial de la Salud (OMS, 2018), que establece las siguientes categorías: bajo peso (IMC < 18.5), normopeso (IMC: $18.5-24.9$ ), sobrepeso (IMC: 25 - 29.9) y obesidad (IMC > 30).

A los estudiantes que presentaron bajo peso, sobrepeso y obesidad se les incluyó en el grupo de casos y a los alumnos con normopeso en el grupo control.

El criterio utilizado para determinar el rendimiento académico de los alumnos fue la calificación promedio obtenida de los cursos al final del estudio, y se clasificó en tres categorías: Excelente/Muy Bueno (90-100), Bueno (80-89) y Regular/Malo (< 80), además se determinó la situación académica de los jóvenes, y se catalogaron como: titulados, egresados, activos y estudiantes irregulares.

Se consideró, titulado: al alumno que cubrió el plan de estudios y cumplió con los requisitos establecidos por la universidad y obtuvo su título; egresado: alumno con certificado o constancia de estudio que aprobó y acreditó todas las asignaturas del plan de estudios; activo: alumno vigente; irregular: alumno que no logró obtener calificación aprobatoria en periodo extraordinario, debiendo repetir la materia en el ciclo escolar siguiente, esta información se obtuvo a través de la Coordinación de Control Escolar del CUCBA.

Se analizó la relación entre el Índice de Masa Corporal (IMC) y el promedio académico de los alumnos mediante una regresión lineal y el coeficiente de correlación de Pearson, también se utilizaron tablas de contingencia para comparar las diferentes variables de casos y controles. Adicionalmente se realizaron pruebas de media y desviación estándar y se calculó la probabilidad con la Prueba $t$ de student para muestras independientes entre casos y controles y la Prueba $X^{2}$.

\section{Resultados}

El presente estudio está conformado por 200 estudiantes universitarios de los cuales 97 fueron casos y 103 controles. De este grupo de estudio, 82 (41\%) corresponden al sexo femenino y $118(59 \%)$ al masculino. En la tabla 1 se muestran las características generales (edad, peso, estatura, IMC, número de cursos reprobados y número de semestres cursados) de los casos y controles.

Tabla 1. Características generales de casos y controles

\begin{tabular}{lcc}
\hline \multicolumn{1}{c}{ Característica } & $\begin{array}{c}\text { Casos }(\mathbf{n = 9 7 )} \\
\text { Media } \pm \mathrm{DE}\end{array}$ & $\begin{array}{c}\text { Controles } \\
(\mathbf{n}=103) \\
\text { Media } \pm \mathrm{DE}\end{array}$ \\
\hline Edad (años) & $21.22 \pm 2.6$ & $21.56 \pm 3.0$ \\
Peso $(\mathrm{kg})^{*}$ & $76.41 \pm 19.7$ & $62.43 \pm 8.8$ \\
Estatura $(\mathrm{cm})^{*}$ & $172 \pm 8.8$ & $168.76 \pm 8.6$ \\
IMC* & $25.62 \pm 5.6$ & $21.87 \pm 1.9$ \\
No. de cursos reprobados & $8.89 \pm 13.7$ & $7.02 \pm 12.3$ \\
Semestres cursados & $9.87 \pm 2.1$ & $9.64 \pm 2.0$ \\
\hline Fuente directa & & \\
DE: Desviación estándar & & \\
$*$ p $<0.05$ & &
\end{tabular}

Al comparar los casos y controles en relación a su situación académica, se observó una menor proporción $(32 \%)$ de alumnos titulados en el grupo de casos comparado con el $37.9 \%$ del grupo control, sin observarse diferencia estadística $(\mathrm{p}>0.05)$ (Tabla 2).

Tabla 2. Situación académica de los alumnos del CUCBA

\begin{tabular}{lcccc}
\hline & $\begin{array}{c}\text { Casos } \\
\mathbf{n = 9 7}\end{array}$ & \multicolumn{3}{c}{$\begin{array}{c}\text { Controles } \\
\mathbf{n = 1 0 3}\end{array}$} \\
\hline T & $\mathrm{n}$ & $(\%)$ & $\mathrm{n}$ & $(\%)$ \\
\hline Titulado & 31 & 32 & 39 & 37.9 \\
Egresado & 47 & 48.4 & 42 & 40.7 \\
Activo & 7 & 7.2 & 10 & 9.7 \\
Irregulares & 12 & 12.4 & 12 & 11.7 \\
\hline Fuente Directa & & & &
\end{tabular}

$\mathrm{Al}$ analizar el rendimiento académico y la situación académica del total de la población estudiada, se observó que los alumnos que tuvieron un rendimiento Excelente/Muy Bueno durante su estancia universitaria, lograron un mayor índice de titulación (64.9\%), mientras que los estudiantes que mostraron un rendimiento académico Regular/Malo $(45.9 \%)$ presentaron una situación académica 
irregular (alumnos que repitieron materias) con diferencia estadística significativa $(\mathrm{p}<0.001)$ (Tabla 3 ). Además, del total de los alumnos que participaron en el estudio, 35\% obtuvieron el título universitario, de éstos el $57.1 \%$ fueron hombres y el $42.9 \%$ mujeres (Tabla 4).

Tabla 3. Rendimiento académico y situación académica de los alumnos del CUCBA

\begin{tabular}{lcccccc}
\hline \multicolumn{3}{c}{ Rendimiento Académico } \\
\hline Alumnos & $\begin{array}{c}\text { Excelente) } \\
\text { Muy Bueno } \\
(\mathbf{n = 3 7 )}\end{array}$ & $\begin{array}{c}\text { Bueno } \\
(\mathbf{n}=\mathbf{1 2 6})\end{array}$ & $\begin{array}{c}\text { Regular/Malo } \\
(\mathbf{n}=\mathbf{3 7})\end{array}$ \\
\hline Titulados & $\mathrm{n}$ & $(\%)$ & $\mathrm{n}$ & $(\%)$ & $\mathrm{n}$ & $(\%)$ \\
Egresados & 24 & 64.9 & 42 & 33.3 & 4 & 10.9 \\
Activos & 11 & 29.7 & 69 & 54.8 & 9 & 24.3 \\
Irregulares & 2 & 5.4 & 8 & 6.3 & 7 & 18.9 \\
\hline Fuente directa & 0 & 0 & 7 & 5.6 & 17 & 45.9 \\
\hline
\end{tabular}

Tabla 4. Porcentaje de alumnos titulados de acuerdo al género

\begin{tabular}{lcccccc}
\multicolumn{8}{c}{ Tabla 4. Porcentaje de alumnos titulados de acuerdo al género } \\
\hline Alumnos & \multicolumn{1}{c}{ Mujeres $(\mathbf{n}=\mathbf{8 2})$} & \multicolumn{1}{c}{ Hombres $(\mathbf{n}=\mathbf{1 1 8})$} & \multicolumn{2}{c}{ Total $(\mathbf{n}=\mathbf{2 0 0})$} \\
\hline Titulados & $\mathrm{n}$ & $(\%)$ & $\mathrm{n}$ & $(\%)$ & $\mathrm{n}$ & $(\%)$ \\
No titulados & 30 & 42.9 & 40 & 57.1 & 70 & 35 \\
\hline Fuente directa & & 40 & 78 & 60 & 130 & 65 \\
\hline
\end{tabular}

Mediante la prueba X2, se comparó el rendimiento académico por género, observándose una mayor proporción de mujeres $(62.2 \%)$ en la categoría Excelente/Muy Bueno, mientras que en los hombres es mayor el porcentaje $(81.1 \%)$ en la categoría de rendimiento académico Regular/Malo con diferencia estadística $\mathrm{p}<0.001$ (Figura 1).

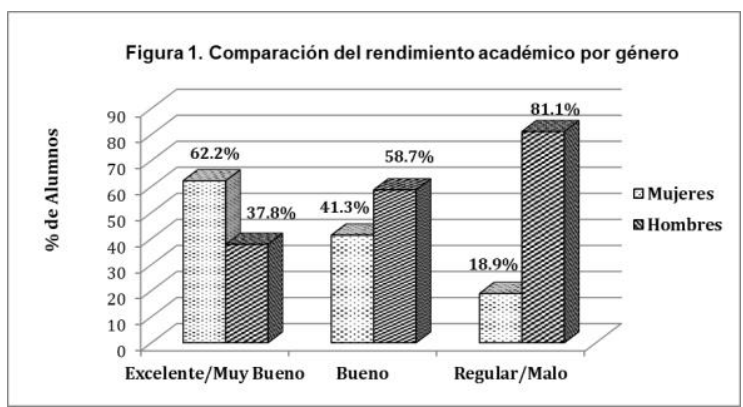

Al determinar el IMC de los estudiantes, el grupo de casos mostró los siguientes resultados: $13 \%$ tuvieron bajo peso (IMC < 18.5), con mayor prevalencia en el sexo femenino, $26.5 \%$ y $9 \%$ presentaron sobrepeso (IMC de 25 - 29.9) y obesidad (IMC $\geq 30$ ) respectivamente, encontrándose en estos dos últimos una mayor prevalencia en el sexo masculino, mientras que el grupo control (IMC $\geq 18.5-24.9)$ estuvo conformado por el $51.5 \%$.

En la tabla 5 se describe la relación entre el rendimiento académico y el IMC de los casos y controles, donde se muestra que en el grupo de estudiantes con sobrepeso existe un porcentaje significativamente mayor $(\mathrm{p}<0.05)$ de alumnos en la categoría Regular/Malo (40.5\%) comparado con los alumnos de la categoría Excelente/Muy Bueno (13.5\%), cabe destacar que en el grupo control un mayor porcentaje de alumnos $(67.6 \%)$ se encuentra en la categoría Excelente/Muy Bueno, aunque sin diferencia estadística.

\begin{tabular}{|c|c|c|c|c|c|c|c|c|c|}
\hline & \multirow[b]{3}{*}{ IMC } & \multicolumn{8}{|c|}{ Rendimiento Académico } \\
\hline & & \multicolumn{2}{|c|}{$\begin{array}{l}\text { Excelente/Muy Bueno } \\
\text { (90 a 100) }\end{array}$} & \multicolumn{2}{|c|}{ Bueno (80 a 89) } & \multicolumn{2}{|c|}{ Regular/Malo $(<80)$} & \multicolumn{2}{|c|}{ Total } \\
\hline & & $n$ & (\%) & $n$ & $(\%)$ & $n$ & $(\%)$ & $n$ & $(\%)$ \\
\hline \multicolumn{10}{|l|}{ Casos } \\
\hline Bajo peso & (<18.5) & $5 a$ & 13.5 & $18 \mathrm{a}$ & 14.3 & $3 a$ & 8.1 & 26 & 13 \\
\hline Sobrepeso & $(25-29.9)$ & $5 a$ & 13.5 & $33 a, b$ & 26.2 & $15 \mathrm{~b}$ & 40.5 & 53 & 26.5 \\
\hline Obesidad & $(\geq 30)$ & $5 \mathrm{a}$ & 5.4 & $14 \mathrm{a}$ & 11.1 & $2 a$ & 5.4 & 18 & 9 \\
\hline \multicolumn{10}{|l|}{ Controles } \\
\hline Normopeso & $(18.5-24.9)$ & 25 a & 67.6 & $61 a$ & 48.4 & $17 \mathrm{a}$ & 45.9 & 103 & 51.5 \\
\hline Total & & 37 & 100 & 126 & 100 & 37 & 100 & 200 & 100 \\
\hline
\end{tabular}

En la figura 2, se muestra la relación entre el IMC y el promedio académico de los casos y controles, ésta presenta una correlación negativa $(\mathrm{r}=-0.216)$ y significativa $(p=0.002)$ entre ambos, la pendiente de la ecuación de regresión de -0.268 implica que por cada unidad que aumenta el IMC, la calificación promedio disminuye 0.268 puntos.

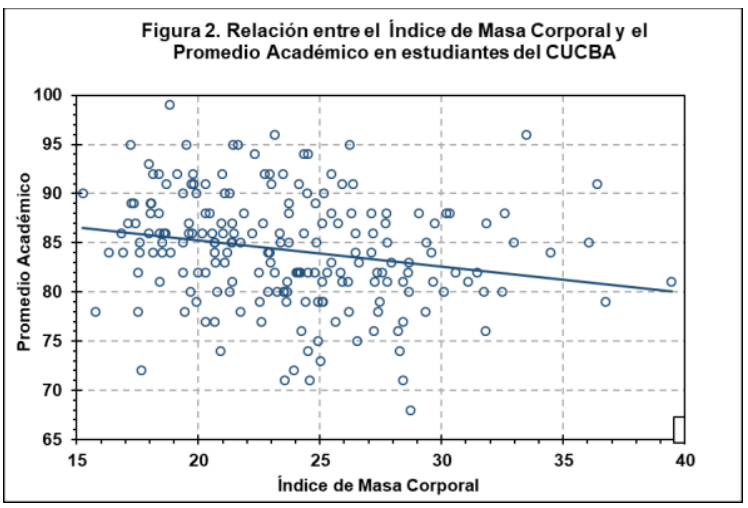

\section{Discusión}

Los estudiantes universitarios se encuentran en un proceso de formación en donde ciertos problemas pueden alterar el equilibrio saludable, afectar el 
rendimiento académico y su proyecto de vida (Ríos y Osornio, 2014).

Al comparar las características generales de los casos y controles, se observaron diferencias estadísticas significativas para peso, estatura e IMC, lo que era de esperarse dado que los estudiantes están clasificados por el IMC, en cuanto al número de cursos reprobados y semestres cursados, no se encontró diferencia.

El rendimiento académico generalmente medido a través de un promedio escolar, expresa el grado de logro que han tenido los estudiantes en la asimilación y comprensión de los contenidos docentes y puede definir el éxito o fracaso en el estudio (García, López de Castro y Rivero, 2014), por otra parte la titulación representa el punto culminante de un proceso de formación en el que los estudiantes adquieren los conocimientos, las habilidades, las aptitudes y las actitudes que les permitirán ejercer con pertinencia la profesión para la cual se formaron, y continuar aprendiendo a lo largo de toda la vida (Mendieta, 2014). Los resultados de esta investigación, mostraron que los alumnos que presentaron un mejor rendimiento académico lograron un mayor índice de titulación.

En el presente estudio los resultados mostraron que un mayor porcentaje $(57.1 \%)$ de hombres lograron obtener un título universitario datos similares $(58,5 \%)$ a lo reportado por Persoglia, Carella y Solari (2017), lo que contrasta con estudios realizados por Meijer y Navarro (2009) donde señalan que las mujeres logran un mejor desempeño en relación a la permanencia y a la titulación.

Se han llevado a cabo múltiples estudios acerca de las diferencias en los procesos intelectuales entre el hombre y la mujer, los que incluyen el rendimiento académico (Echavarri, Godoy y Olaz, 2007). En nuestra investigación se encontró que una mayor proporción de mujeres obtuvo un rendimiento académico Excelente/Muy Bueno respecto a los hombres, resultados similares a lo reportado por Sepúlveda et al., (2011), Persoglia, Carella y Solari (2017) y Gómez, Oviedo y Martínez (2011). Algunos estudios sugieren que un mejor rendimiento académico en mujeres, podría explicarse entre otros aspectos al tipo de evaluaciones en las que se requiere del uso importante del léxico, además reflexionan más que los hombres sobre cómo preparar la información para un examen oral o escrito, en aspectos tales como asociación libre, redacción y/o presentación (Sepúlveda et al., 2011), sin embargo los resultados de Sepúlveda, Montero, Pérez, Contreras y Solar (2010) muestran que no existen diferencias significativas que se asocie con el género, al respecto se destaca que el rendimiento académico estaría relacionado con el estilo de aprendizaje y el empleo de estrategias de aprendizaje (Yip, 2012).

Al comparar el rendimiento académico de los casos y controles, en el grupo de casos, los alumnos con sobrepeso presentaron un rendimiento académico significativamente menor $(\mathrm{p}<0.05)$, lo que concuerda con Anderson y Good (2017) quienes encontraron que los estudiantes con sobrepeso y obesidad tuvieron una calificación más baja que los alumnos con normopeso, Correa-Burrows, Burrows, Blanco, Reyes y Gahagan (2016) consideran que el consumo excesivo de alimentos altamente energéticos, bajos en fibra y con elevadas cantidades de grasa (que contribuyen al sobrepeso y obesidad) está relacionado con un bajo rendimiento académico.

Los resultados de los indicadores antropométricos del presente estudio, particularmente en lo relacionado al sobrepeso (26.5\%) y obesidad (9\%), difieren de Zea, Vargas, Nieva y Anaya (2016), quienes encontraron que, de los alumnos ingresantes a la Facultad de Enfermería en Perú, un 54.8\% presentó un IMC dentro de los valores normales, $19.4 \%$ con bajo peso, $9.7 \%$ con sobrepeso y un $16.1 \%$ con obesidad. Respecto al bajo peso, nuestros resultados muestran una mayor prevalencia en mujeres, datos que son diferentes a lo reportado por Pérez-Cueto y Eulert (2009) donde la prevalencia de bajo peso fue insignificante y afectó principalmente a los varones, y coinciden con Ríos y Osornio (2014), los cuales mencionan que el sobrepeso y la obesidad pueden deberse entre otros factores a los estilos alimentarios de los jóvenes universitarios caracterizados por saltarse comidas, comer cualquier cosa para saciar el hambre o el consumo de comida rápida.

Investigaciones realizadas en estudiantes universitarios permiten pensar en la existencia de un estado nutricional inadecuado en este grupo poblacional, que puede ser un componente que incide 
en el aprendizaje de los estudiantes (Fleitas, García y Zambrano, 2015). Aunque la correlación en esta investigación, entre el IMC y el rendimiento académico fue débil, indica que a medida que el IMC de los estudiantes se incrementa, su calificación promedio disminuye, esto difiere de lo reportado por Wehigaldeniya, Oshani y Kumara (2017) y Zea, Vargas, Nieva y Anaya (2016), quienes refieren que no existe asociación entre ambos, sin embargo nuestros datos son consistentes con otros estudios que han demostrado una asociación significativa entre el IMC y el rendimiento académico (Anderson y Good, 2017), existe evidencia que dietas poco saludables, la baja ingesta de frutas y verduras y la insuficiente actividad física son variables que pudieran estar relacionadas con el bajo rendimiento académico (Correa-Burrows, Burrows, Blanco, Reyes y Gahagan, 2016; Anderson y Good, 2017).

\section{Conclusiones}

La correlación débil encontrada entre el IMC y el rendimiento académico en esta investigación, sugiere involucrar en estudios posteriores otras variables como frecuencia de consumo de alimentos, actividad física, hábitos de sueño, estrategias de aprendizaje, y factores socioeconómicos entre otros.

\section{Bibliografía}

Anderson, A., y Good, D. (2017). Increased body weight affects academic performance in university students. Preventive Medicine Reports, 5, 220-223. Recuperado de

https://www.ncbi.nlm.nih.gov/pmc/articles/PMC5222 954/

Antonella, P., Vidal, P., Brassesco, B., Viola, L., \& Aballay, L. (2015). Estado nutricional en estudiantes universitarios: su relación con el número de ingestas alimentarias diarias y el consumo de macronutrientes. Nutrición Hospitalaria, 31(4), 1748-1756. Recuperado de https://dx.doi.org/10.3305/nh.2015.31.4.8399

Arroyo, M.., Rocandio, P., Ansotegui, L., Apalauza, E., Salces, I., \& Rebato, E. (2006). Calidad de la dieta, sobrepeso y obesidad en estudiantes universitarios. Nutrición Hospitalaria, 21(6), 673-679. Recuperado de

http://scielo.isciii.es/scielo.php?script=sci_arttext\&pi $\mathrm{d}=$ S0212-16112006000900007\&lng=es\&tlng=es.

Correa-Burrows, P., Burrows, R., Blanco, E., Reyes, M., \& Gahagan, S. (2016). Nutritional quality of diet and academic performance in Chilean students. Bull
World Health Organ, 94, 185-192. Recuperado de https://www.ncbi.nlm.nih.gov/pmc/articles/PMC4773 934/pdf/BLT.15.161315.pdf.

Echavarri, M., Godoy, J.C., y Olaz, F. (2007). Diferencias de género en habilidades cognitivas y rendimiento académico en estudiantes universitarios. Universitas Psychologica, 6(2), 319-329. Recuperado de http://pepsic.bvsalud.org/pdf/up/v6n2/v6n2a11.pdf.

ENSANUT MC. Encuesta Nacional de Salud y Nutrición Medio Camino. (2016). Informe final de resultados. Recuperado de https://www.gob.mx/cms/uploads/attachment/file/209 093/ENSANUT.pdf

Fleitas, D., García, E. L, y Zambrano, D. T. (2015). Estado nutricional y rendimiento académico en estudiantes de la Facultad de Enfermería de la Universidad Laica Eloy Alfaro de Manabí. Revista Electrónica Formación y Calidad Educativa (REFCalE), 3(3), 43-58. Recuperado de http://runachayecuador.com/refcale/index.php/refcale/ article/view/463

García, Y., López de Castro, D., y Rivero, O. (2014). Estudiantes universitarios con bajo rendimiento académico, ¿qué hacer?. EDUMECENTRO, 6(2), 272$278 . \quad$ Recuperado de http://scielo.sld.cu/pdf/edu/v6n2/edu18214.pdf.

García, M., Padrón, J., Ortiz-Hernández, L., Camacho, M., y Vargas, R. (2005). Efecto de la desnutrición sobre el desempeño académico de escolares. Revista Mexicana de Pediatría, 72(3), 117-125. Recuperado de http://www.medigraphic.com/pdfs/pediat/sp2005/sp053d.pdf

Ghosh, D., Rakshit, S., y Bhattacharya, M. (2013). Academic Performance and Nutritional Status - A Case Study on College Students in North Tripura. Journal of Research \& Method in Education, 1(4), 5768. Recuperado de http://www.iosrjournals.org/iosrjrme/papers/Vol-1\%20Issue-4/I0145768.pdf

Gómez, D., Oviedo, R., y Martínez, E. (2011). Factores que influyen en el rendimiento académico del estudiante universitario. Tecnociencia, 5(2), 90-97. Recuperado de http://tecnociencia.uach.mx/numeros/v5n2/data/Facto res_que_influyen_en_el_rendimiento_academico_del _estudiante_universitario.pdf

Jiménez, M., y Madrigal, H. (2009). Evaluación del estado nutricional de los alumnos de la Escuela Preparatoria de ULSA (D.F). Revista del Centro de Investigación. 
Universidad La Salle, 8(31), 35-50. Recuperado de http://www.redalyc.org/articulo.oa?id=34211305003.

Jukes, M., Mcguire, J., Method, F. \& Sternberg, R. (2002). "Nutrición y educación". En Nutrición: La base para el desarrollo. Ginebra: SCN. Recuperado de: http://cienciaysalud.laverdad.es/lanutricionesconcienc ia/01-

Los\%20fundamentos/Complementario/NutricionBase Desarrollo.pdf

Mendieta, R, A. (2014). Propuesta: Dirección General de Titulación. En Perspectiva de los retos: Innovando juntos. Ingreso, permanencia, egreso y titulación en el nivel superior (11-19). México. Ediciones del Lirio. Recuperado de http://www.dgpi.buap.mx/obracolegiada/Vol\%20IV\% 20Ingreso,\%20permanencia,\%20egreso $\% 20 \mathrm{y} \% 20$ titul acion $\% 20 \mathrm{en} \% 20 \mathrm{el} \% 20$ nivel\%20superior.pdf

Meijer, W., y Navarro, Y. (2009). Egresados universitarios y la equidad de género: evidencias de una exitosa revolución silenciosa. X Congreso Nacional de Investigación Educativa. Recuperado de http://www.comie.org.mx/congreso/memoriaelectroni ca/v10/pdf/area_tematica_10/ponencias/1583-F.pdf

Pérez-Cueto y Eulert, M.E. (2009). Estado nutricional de un grupo de estudiantes Universitarios de $\mathrm{La} \mathrm{Paz}$, Bolivia. Nutrición Hospitalaria, 24 (4), 511. Recuperado de http://scielo.isciii.es/pdf/nh/v24n4/carta_cientifica1.p df

Persoglia, L. M., Carella, L., y Solari, E. (2017). Rendimiento académico y características socioeconómicas de graduados. Ciencia, Docencia y Tecnología, 28(54), 235-251. Recuperado de http://www.redalyc.org/pdf/145/14551170009.pdf.

Ríos, R., y Osornio, L. (2014). Perfil emocional, obesidad percibida vs IMC y rendimiento académico en estudiantes universitarios. Alternativas en Psicología, 17(29), 150-164. Recuperado de http://pepsic.bvsalud.org/pdf/alpsi/v17n29/n29a10.pdf

Sánchez-Ojeda, M. A y De Luna-Bertos, E. (2015). Hábitos de vida saludable en la población universitaria. Nutr Hosp. 31(5), 1910-1919. Recuperado de http://www.aulamedica.es/nh/pdf/8608.pdf

Sepúlveda, M. J., Montero E. F., Pérez, R., Contreras, E., Solar, M. I. (2010). Diferencias de género en el perfil de estilos y del uso de estrategias cognitivas de aprendizaje de estudiantes de farmacología. Revista Estilos de Aprendizaje. 5(05), 1-21. Recuperado de https://www2.uned.es/revistaestilosdeaprendizaje/nu mero_5/articulos/lsr_5_articulo_5.pdf
Sepúlveda, M.J., López, M., Torres, P., Luengo, J., Montero, E., y Contreras, E. (2011). Diferencias de género en el rendimiento académico y en el perfil de estilos y de estrategias de aprendizaje en estudiantes de Química y Farmacia de la Universidad de Concepción. Revista Estilos de Aprendizaje, 4(7), 29-45. Recuperado de http://redined.mecd.gob.es/xmlui/bitstream/handle/11 162/81523/00820113012832.pdf?sequence $=1$

OMS. Organización Mundial de la Salud. (2018). Recuperado de http://www.who.int/es/news-room/factsheets/detail/obesity-and-overweight.

Wehigaldeniya, D., Oshani, P., \& Kumara, I. (2017). Height, Weight, Body Mass Index (BMI) and Academic Performance (AP) of University Students in Sri Lanka: With Special Reference to the University of Kelaniya. International Journal of Scientific and Research Publications, 7(2), 217-219. Recuperado de https://www.researchgate.net/publication/324273948_ Height_Weight_Body_Mass_Index_BMI_and_Acade mic_Performance_AP_of_University_Students_in_Sr i_Lanka_With_Special_Reference_to_the_University _of_Kelaniya.

Yip, M. (2012) Learning strategies and self-efficacy as predictors of academic performance: a preliminary study, Quality in Higher Education, 18:1, 23-34. Recuperado

de https://www.tandfonline.com/doi/pdf/10.1080/135383 22.2012.667263 ?needAccess $=$ true

Zea, C. C., Vargas, A., Nieva, L., y Anaya, R. (2016). "Estado nutricional y rendimiento académico de los ingresantes a la Facultad de Enfermería de una Universidad Pública”. Ágora Revista Científica, 03(01), 257-264. Recuperado de http://www.revistaagora.com/index.php/cieUMA/artic le/view/49. 